\title{
RESONANCES FOR SCHRÖDINGER OPERATORS WITH COMPACTLY SUPPORTED POTENTIALS
}

\author{
T. J. CHRISTIANSEN AND P. D. HISLOP
}

\begin{abstract}
We describe the generic behavior of the resonance counting function for a Schrödinger operator with a bounded, compactlysupported real or complex valued potential in $d \geq 1$ dimensions. This note contains a sketch of the proof of our main results [5, 6] that generically the order of growth of the resonance counting function is the maximal value $d$ in the odd dimensional case, and that it is the maximal value $d$ on each nonphysical sheet of the logarithmic Riemann surface in the even dimensional case. We include a review of previous results concerning the resonance counting functions for Schrödinger operators with compactly-supported potentials.
\end{abstract}

\section{Introduction and Survey of Results}

We are interested in the resonance counting function for Schrödinger operators $H_{V}=-\Delta+V$, acting on $L^{2}\left(\mathbb{R}^{d}\right)$, with compactly supported realor complex-valued potentials $V \in L_{0}^{\infty}\left(\mathbb{R}^{d}\right)$. We give a survey of results and discuss on our recent results on the generic behavior of the resonance counting function [5] and [6]. We refer the reader to [3, 5, 6] for complete proofs of Theorems 1 and 2 .

Resonances are the poles of the meromorphic continuation of the cutoff resolvent of $H_{V}$ as we now explain. For any $V \in L_{0}^{\infty}\left(\mathbb{R}^{d}\right)$, we denote by $\chi_{V} \in C_{0}^{\infty}\left(\mathbb{R}^{d}\right)$ any smooth, compactly supported function such that $\chi_{V} V=V$. The cut-off resolvent for the Laplacian $H_{0}=-\Delta$ is defined to be $\mathcal{R}_{0}(\lambda) \equiv \chi_{V}\left(H_{0}-\lambda^{2}\right)^{-1} \chi_{V}$. This is a holomorphic, bounded operatorvalued function on $L^{2}\left(\mathbb{R}^{d}\right)$ for $\lambda \in \mathbb{C}$ for $d \geq 1$ odd, and for $\lambda \in \Lambda$, for $d \geq 4$ even. For $d=2$ it is holomorphic on $\Lambda \backslash\{0\}$, with a logarithmic singularity at $\lambda=0$. The unperturbed cut-off resolvent $\mathcal{R}_{0}(\lambda)$ has the representation

$$
\mathcal{R}_{0}(\lambda)=E_{1}(\lambda)+\left(\lambda^{d-2} \log \lambda\right) E_{2}(\lambda),
$$

where $E_{1}(\lambda)$ and $E_{2}(\lambda)$ are entire operator-valued functions with $E_{2}(\lambda)=0$ for $d \geq 1$ odd, and for $d=2$, the operator $E_{2}(\lambda=0)$ is a finite-rank operator This follows, for example, from the formula for the Green's function for the Laplacian

$$
R_{0}(\lambda)=\frac{i}{4}\left(\frac{\lambda}{2 \pi|x-y|}\right)^{(d-2) / 2} H_{(d-2) / 2}^{(1)}(\lambda|x-y|),
$$


where the Hankel function of the first kind is defined by $H_{\nu}^{(1)}(z)=J_{\nu}(z)+$ $i N_{\nu}(z)$, and the expansion of the Neumann Bessel function $N_{\nu}(z)$, when $\nu \in \mathbb{N}$.

An application of the second resolvent formula allows us to write the perturbed, cut-off resolvent $\mathcal{R}_{V}(\lambda)$ for $H_{V}$ as

$$
\mathcal{R}_{V}(\lambda)\left(1+V \mathcal{R}_{0}(\lambda)\right)=\mathcal{R}_{0}(\lambda)
$$

The resolvent $R_{V}(\lambda)$ is analytic for $\operatorname{Im} \lambda>>0$, and it is meromorphic for $\operatorname{Im} \lambda>0$ with at most finitely-many poles with finite multiplicities corresponding to the eigenvalues of $H_{V}$. Thanks to (3), the perturbed, cut-off resolvent $\mathcal{R}_{V}(\lambda)$ extends as a meromorphic, bounded operator-valued function on $\mathbb{C}$ if $d \geq 1$ is odd, and onto the Riemannn surface $\Lambda$ if $\mathrm{d} \geq 4$ is even, with an additional logarithmic singularity at $\lambda=0$ when $d=2$. The poles of these continuations are the resonances of $H_{V}$. They are independent of the choice of $\chi_{V}$ satisfying the above conditions. It is seen that they correspond to the values of $\lambda$ for which $1+V \mathcal{R}_{0}(\lambda)$ is not boundedly invertible. We will use this fact in section 2.1.

We will always assume $V \in L_{0}^{\infty}\left(\mathbb{R}^{d}\right)$. The resonance counting function $n_{V}(r)$ for $d \geq 1$ and odd is defined to be the number of poles $\lambda_{j}$ of $\mathcal{R}_{V}(\lambda)$, including multiplicities, with $\left|\lambda_{j}\right| \leq r$ and $\operatorname{Im} \lambda_{j}<0$. For $d \geq 2$ even, the resonance counting function $n_{V, m}(r)$ for the $m^{t h}$-sheet $\Lambda_{m}, m \neq 0$, is defined as the number of poles $\lambda_{j}$ of $\mathcal{R}_{V}(\lambda)$ with $\left|\lambda_{j}\right| \leq r$ and $m \pi<\arg \lambda_{j}<$ $(m+1) \pi$.

The best results on the asymptotic behavior of $n_{V}(r)$ is due to Zworski [42] for $d=1$. He proved that

$$
n_{V}(r)=\frac{2}{\pi}|\operatorname{supp} V| r+o(r) .
$$

He obtained a similar asymptotic expansion in $\mathbb{R}^{d}, d \geq 3$ odd, for a family of spherically symmetric potentials $V(r)$ with compact support $[0, a]$ and a discontinuity at $r=a$ [43]. The exact form of the constant of the leading term in this expansion was identified by Stefanov [33. An asymptotic expansion of the form (4) for a class of super-exponentially decaying potentials in one-dimension was proved by Froese [7] and Simon [28]. In what follows, we consider dimensions $d \geq 2$.

We mention that many results are known for the resonance counting function for Schrödinger operators $H_{V}(h)=-h^{2} \Delta+V$ in the semiclassical regime of small $h>0$, but we will not discuss these here. We refer to the extensive papers of Sjöstrand [31] and of Sjöstrand and Zworski [32] for the details and references. We also refer the reader to two review articles [45. 38. Many aspects of resonances for Schrödinger operators were explored by physicists. Regge [21] studied the Jost function and proved that there are infinitely many resonances for compactly supported, real-valued potentials. This and other aspects of resonances are discussed by Newton [15. Nussenzveig [16] studied analytically and numerically the poles of the 
$S$-matrix for square well and square barrier potentials of the type described in section 6 ,

1.1. Upper Bounds. In analogy with the celebrated Weyl upper bound for the eigenvalue counting function for elliptic operators on compact manifolds, upper bounds for the resonance counting function were proven in the eighties and nineties. Because the meromorphic continuation of the cut-off resolvent depends on the parity of the dimension, the results are different for odd and even dimensions. The upper bounds hold for both complex and real-valued potentials.

1.1.1. Upper bounds - odd dimensions $d \geq 3$. Polynomial upper bounds on the resonance counting function $n_{V}(r)$ were first proved by Melrose [13]. He showed that $n_{V}(r) \leq C_{d}\langle r\rangle^{d+1}$. This upper bound was improved by Zworski [41] who obtained the optimal upper bound $n_{V}(r) \leq C_{d}\langle r\rangle^{d}$. Another proof was given by Vodev [35] and by Sjöstrand and Zworski [32. The optimal constant $C_{d}$ was computed by Stefanov [33].

1.1.2. Upper bounds - even dimensions $d \geq 2$. The first upper bounds were proven by Intissar [9]. Intissar defined, for any $\epsilon>0$ and $r>1$, a resonance counting function $N_{I n}(\epsilon, r) \equiv\left\{\lambda_{j}\left|r^{-\epsilon}<\right| \lambda_{j}\left|<r^{\epsilon},\right| \arg \lambda_{j} \mid<\epsilon \log r\right\}$. For even dimensions $d \geq 4$, and for any $\epsilon \in(0, \sqrt{2} / 2)$, he proved the polynomial upper bound $N_{I n}(\epsilon, r) \leq C_{\epsilon}\langle r\rangle^{d+1}$. Vodev considered the resonance counting function $N_{V o}(r, a)$ defined as the number of resonances $\lambda_{j}$ satisfying $0<$ $\left|\lambda_{j}\right|<r$ and $\left|\arg \lambda_{j}\right|<a$, for $r, a>1$. This function counts the number of resonances in a fixed sector of opening angle $a$. Vodev proved the upper bound for even dimensions $d \geq 2$ [36, 37]:

$$
N_{V o}(r, a) \leq C_{d} a\left(r^{d}+(\log a)^{d}\right) .
$$

Note the explicit dependance of the coefficient on $a$. Since the Green's function has a logarithmic singularity at zero energy in the two-dimensional case, this required a separate argument. However, this upper bound does not distinguish between the resonances that might occur on each sheet $\Lambda_{m}, m \in$ $\mathbb{Z}^{*} \equiv \mathbb{Z} \backslash\{0\}$

1.2. Lower bounds. There are few lower bounds in dimensions $d \geq 2$. As above, there are different bounds depending upon whether the dimension is even or odd. Because of the existence of complex potentials with no resonances [4], these results hold only for nontrivial real-valued potentials.

1.2.1. Lower bounds - odd dimensions $d \geq 3$. The best known lower bounds for odd dimensions, other than the asymptotic results for certain radial potentials [43], hold for potentials $V \in L_{0}^{\infty}\left(\mathbb{R}^{d}\right)$ of fixed sign. In the odd dimensional case, Lax and Phillips [10] proved that if $V \geq 0$ is bounded below by a characteristic function on a ball of radius $R>0$, then that the number of purely imaginary poles in the lower half complex plane of modulus less or equal to $r>0$ is bounded from below by $c_{d} r^{d-1}$, with 
$c_{d}>0$. Menzala and Schonbek [12] allow potentials with a small negative component so that $-\Delta+V>0$ in the quadratic form sense. Vasy 34] proved the existence of an infinite number of purely imaginary poles when $\pm V \geq 0$, extending [10 to the case of a strictly negative potential. These results imply that $n_{V}(r)$ is similarly bounded from below. The idea of these proofs is to note that the scattering amplitude is a monotone function of $V$ at purely imaginary energies. This reduces the problem to an explicit calculation of the number of purely imaginary poles for the symmetric step potential $V(x)=V_{0} \chi_{B_{R}(0)}(|x|)$, for any $V_{0}>0$, where $B_{R}(x)$ is the ball of radius $R>0$ centered at $x$.

In the general case of nontrivial, smooth, real-valued $V \in C_{0}^{\infty}\left(\mathbb{R}^{d}\right)$, Melrose [14] observed from the Poisson formula that there must be infinitelymany resonances if any of the coefficients in the expansion of the heat kernel for $k>d-2$ are nonvanishing. In particular, for $d=3$, there are an infinite number of resonances since $a_{1}=\int V^{2} \neq 2$. This was extended to a class of super-exponentially decaying, smooth, real-valued potentials in [1, 25, 26. The first quantitative lower bounds for the resonance counting function for nontrivial, smooth, real-valued $V \in C_{0}^{\infty}\left(\mathbb{R}^{d}\right)$, not of fixed sign, were proved in 2]. In particular, it was proved there that

$$
\limsup _{r \rightarrow \infty} \frac{n_{V}(r)}{r(\log r)^{-p}}=\infty,
$$

for all $p>1$. For the same family of potentials, Sá Barreto [22] improved this to

$$
\limsup _{r \rightarrow \infty} \frac{n_{V}(r)}{r}>0 .
$$

We mention that, in particular, all these lower bounds require the potential to be smooth.

1.2.2. Lower bounds - even dimensions $d \geq 2$. There are only two results on lower bounds in the even dimensional case for $d \geq 4$. Sá Barreto and Tang [24] proved the existence of at least one resonance for a real-valued, compactly-supported, smooth nontrivial potential. Sá Barreto [23] studied the resonance counting function $N_{S a B}(r)$ defined to be the number of resonances $\lambda_{j}$ with $1 / r<\left|\lambda_{j}\right|<r$ and $\left|\arg \lambda_{j}\right|<\log r$. As $r \rightarrow \infty$, this region in the Riemann surface $\Lambda$ opens like $\log r$. Sá Barreto proved that for even $d \geq 4$,

$$
\limsup _{r \rightarrow \infty} \frac{N_{S a B}(r)}{(\log r)(\log \log r)^{-p}}=\infty
$$

for all $p>1$.

1.3. Generic behavior. The order of growth of a positive, real-valued, monotone increasing function $n(r)$ is defined by

$$
\rho \equiv \limsup _{r \rightarrow \infty} \frac{\log n(r)}{\log r},
$$


when it is finite. Roughly speaking, we prove that the order of growth of the resonance counting function is maximal for most real-valued or complexvalued potentials in $L_{0}^{\infty}\left(\mathbb{R}^{d}\right)$ in the even and odd dimensional cases. Here, the term "most potentials" is meant in the Borel sense. Let $X$ be a metric space. A subset is said to be Baire typical or generic if it is a dense $G_{\delta}$ subset of $X$.

We refer to the beginning of section 1 for the definition of the resonance counting function $n_{V}(r)$ in odd dimensions, and of $n_{V, m}(r)$ for even dimensions. The order of growth of the resonance counting function $n_{V}(r)$ or $n_{V, m}(r)$ is defined by

$$
\rho_{V} \equiv \limsup _{r \rightarrow \infty} \frac{\log n_{V}(r)}{\log r}, d \text { odd }
$$

or, by

$$
\rho_{V, m} \equiv \limsup _{r \rightarrow \infty} \frac{\log n_{V, m}(r)}{\log r}, d \text { even. }
$$

As discussed above, it is known that both $\rho_{V}$ and $\rho_{V, m}$ are bounded above by $d$. We are interested in the values of these order of growth exponents for generic potentials. The notion of generic used here was employed by Simon [28] in his study of singular continuous spectrum for Schrödinger operators. We proved the following theorem in [5, 6].

Theorem 1. Let $K \subset \mathbb{R}^{d}$ be a fixed, compact set with nonempty interior. There is a dense $G_{\delta}$ set $\mathcal{V}_{F}(K) \subset L_{0}^{\infty}(K ; F)$, for $F=\mathbb{R}$ or $F=\mathbb{C}$, such that if $V \in \mathcal{V}_{F}(K)$, and if $d \geq 2$ is even, then $\rho_{V, m}=d$ for all $m \in \mathbb{Z} \backslash\{0\}$, or, if $d \geq 3$ is odd, then $\rho_{V}=d$.

For odd dimensions, a similar result holds with $C_{0}^{\infty}(K ; F)$ in place of $L_{0}^{\infty}(K ; F)$ 3, 5. For the proof of Theorem 1, it is essential to have an explicit example of a potential for which the order of growth of the resonance counting function is $d$. For odd dimensions, Zworski 43 . proved the asymptotic form of $n_{V}(r)$ for a class of positive, nontrivial, spherically symmetric potentials. This result is, of course, stronger than the lower bound stated in Theorem 2, Since, for our purposes, only a lower bound is required, this gives an alternate, and perhaps simpler, proof. For the even dimensional case, we proved a lower bound on the resonance counting function for spherically symmetric, constant, positive potentials on each nonphysical sheet. Let $B_{R}(x)$ be the ball of radius $R>0$ centered at $x \in \mathbb{R}^{d}$.

Theorem 2. Let $V(x)=V_{0} \chi_{B_{R}(0)}(x)$ for $V_{0}>0$. Then, for $d$ even, there is a constant $c_{m}>0$ so that $n_{V, m}(r) \geq c_{m} r^{m}$, for $m \in \mathbb{Z}^{*}$. For $d$ odd, there is a constant $c_{0}>0$ so that $n_{V}(r)>c_{0} r^{d}$.

In the remainder of this article, we sketch the main ideas behind the proofs of Theorems 1 and 2, Section 2 presents the reduction to a problem of counting zeros of a function holomorphic on a half-plane. We prove Theorem 11in section 3 modulo results concerning plurisubharmonic functions that are 
presented in sections 4 and 5. The proof of Theorem 2 is sketched in section 6.

\section{Reduction to A Zero COUnting PROBlem}

As is typical for these problems, we reduce the estimate on the resonance counting function to one on the number of zeros of a function holomorphic in the half plane $\operatorname{Im} \lambda>0$. In our papers, we took different approaches depending upon whether the dimension is even or odd. Here, we unify the approaches and show how to use the method of [6] for either parity of the dimension. For odd dimensions, in [5] and [3], resonances were treated by considering the $S$-matrix and its scattering phase. Crucial for the argument is the fact that the $S$-matrix admits a Weierstrass product representation in the complex plane. For the even dimensional case, we work directly with the second resolvent formula and we do that here for any dimension.

2.1. Resolvent approach: any dimension. Let $V \in L_{0}^{\infty}\left(\mathbb{R}^{d}\right)$ and let $\chi_{V}$ be any compactly-supported, smooth function $\chi_{V} \in C_{0}^{\infty}\left(\mathbb{R}^{d}\right)$ so that $\chi_{V} V=V$. The cut-off free resolvent $\mathcal{R}_{0}(\lambda) \equiv \chi_{V} R_{0}(\lambda) \chi_{V}$ has a meromorphic continuation to $\Lambda$, the logarithmic cover of the plane, for $d \geq 4$ even, or to $\mathbb{C}$, for $d \geq 1$, odd (see, for example, [14, 27]). For $d=2$ there is a logarithmic singularity at $\lambda=0$. For $d$ odd, there are two distinct sheets, the physical sheet $\Lambda_{0}$, and $\Lambda_{-1}$. For $d$ even, there is the physical sheet and infinitely-many distinct nonphysical sheets $\Lambda_{m}, m \in \mathbb{Z}^{*}$. In order to unify notation, we define $m(d)$ to be equal to $m \in \mathbb{Z}$ when $d$ is even, and, when $d$ is odd, we take $m=-1$ to be the lower-half complex plane, so $m(d)$ is $m \bmod 2$. In this way, the sheets $\Lambda_{2 k}$ are all identified with $\Lambda_{0}$ and the sheets $\Lambda_{2 k+1}$ are all identified with $\Lambda_{-1}$.

We use the following key identity, that follows from (2) and the formulas for the meromorphic continuation of Hankel functions (see [6, section 6] or [17, chapter 7]), relating the free resolvent on $\Lambda_{m}$ to that on $\Lambda_{0}$, for any $m \in \mathbb{Z}$,

$$
R_{0}\left(e^{i m \pi} \lambda\right)=R_{0}(\lambda)-m(d) T(\lambda), \text { where } m(d)=\left\{\begin{array}{lll}
m & \bmod 2 & d \text { odd } \\
m & & d \text { even }
\end{array}\right.
$$

The operator $T(\lambda)$ on $L^{2}\left(\mathbb{R}^{d}\right)$ has a Schwartz kernel

$$
T(\lambda, x, y)=i \pi(2 \pi)^{-d} \lambda^{d-2} \int_{\mathbb{S}^{d-1}} e^{i \lambda(x-y) \cdot \omega} d \omega,
$$

see [14, Section 1.6]. We note that for any $\chi \in C_{0}^{\infty}\left(\mathbb{R}^{d}\right), \chi T(\lambda) \chi$ is a holomorphic trace-class operator for $\lambda \in \mathbb{C}$. The operator $T$ has a kernel proportional to $|x-y|^{(-d+2) / 2} J_{(d-2) / 2}(\lambda|x-y|)$ when $d$ is odd, and to $\mid x-$ $\left.y\right|^{(-d+2) / 2} N_{(d-2) / 2}(\lambda|x-y|)$ when $d$ is even. The different behavior of the free resolvent for $d$ odd or even is encoded in (12).

By the second resolvent formula (3), the poles of $\mathcal{R}_{V}(\lambda)$ with multiplicity, correspond to the zeros of $I+V R_{0}(\lambda) \chi_{V}$. We can reduce the analysis of the 
zeros of the continuation of $I+V R_{0}(\lambda) \chi_{V}$ to $\Lambda_{m}$ to the analysis of zeros of a related operator on $\Lambda_{0}$ using (12). If $0<\arg \lambda<\pi$ and $m \in \mathbb{Z}$, then $e^{i m \pi} \lambda \in \Lambda_{m}$, and

$$
\begin{aligned}
I+V R_{0}\left(e^{i m \pi} \lambda\right) \chi & =I+V\left(R_{0}(\lambda)-m T(\lambda)\right) \chi_{V} \\
& =\left(I+V R_{0}(\lambda) \chi_{V}\right)\left(I-m\left(I+V R_{0}(\lambda) \chi_{V}\right)^{-1} V T(\lambda) \chi_{V}\right) .
\end{aligned}
$$

For any fixed $V \in L_{0}^{\infty}\left(\mathbb{R}^{d}\right)$, there are only finitely many poles of $(I+$ $\left.V R_{0}(\lambda) \chi_{V}\right)^{-1}$ with $0<\arg \lambda<\pi$. Thus

$$
f_{V, m}(\lambda)=\operatorname{det}\left(I-m\left(I+V R_{0}(\lambda) \chi_{V}\right)^{-1} V T(\lambda) \chi_{V}\right)
$$

is a holomorphic function of $\lambda$ when $0<\arg \lambda<\pi$ and $|\lambda|>c_{0}\left\langle\|V\|_{L^{\infty}}\right\rangle$. Moreover, with at most a finite number of exceptions, the zeros of $f_{V, m}(\lambda)$, with $0<\arg \lambda<\pi$ correspond, with multiplicity, to the poles of $\mathcal{R}_{V}(\lambda)$ with $m \pi<\arg \lambda<(m+1) \pi$. Henceforth, we will consider the function $f_{V, m}(\lambda)$, for $m \in \mathbb{Z}^{*} \equiv \mathbb{Z} \backslash\{0\}$, on $\Lambda_{0}$. For $d$ odd, we are only interested in $m=-1$. In this case, the zeros of $f_{V,-1}(\lambda)$, for $\lambda \in \Lambda_{0}$, correspond to the resonances. A similar approach in the $d$ odd case was employed by Froese [7].

2.2. The $S$-matrix approach in odd dimensions. We comment on the case of odd dimensions used in [3, 5]. We denote the scattering matrix for the pair $H_{0}=-\Delta$ and $H_{V}=H_{0}+V$ by $S_{V}(\lambda)$, acting on $L^{2}\left(\mathbb{S}^{d-1}\right)$. In the case that $V$ is real-valued, this is a unitary operator for $\lambda \in \mathbb{R}$. The $S$-matrix is given explicitly by

$$
S_{V}(\lambda)=I+c_{d} \lambda^{d-2} \pi_{\lambda}\left(V-V R_{V}(\lambda) V\right) \pi_{-\lambda}^{t} \equiv I+\mathcal{T}_{\lambda},
$$

where $\pi_{\lambda}: L^{2}\left(\mathbb{R}^{d}\right) \rightarrow L^{2}\left(\mathbb{S}^{d-1}\right)$ is defined by $\left(\pi_{\lambda} f\right)(\omega)=\int e^{-i \lambda x \cdot \omega} f(x) d x$ [40]. Under the assumption that $\operatorname{supp} V$ is compact, the operator $\mathcal{T}_{\lambda}$ : $L^{2}\left(\mathbb{S}^{d-1}\right) \rightarrow L^{2}\left(\mathbb{S}^{d-1}\right)$ is trace class. The $S$-matrix has a meromorphic continuation to the entire complex plane with finitely many poles for $\operatorname{Im} \lambda>0$ corresponding to eigenvalues of $H_{V}$, and resonance poles in $\operatorname{Im} \lambda<0$. We recall that if $\operatorname{Im} \lambda_{0} \geq c_{0}\left\langle\|V\|_{L^{\infty}}\right\rangle$, the multiplicities of $\lambda_{0}$, as a zero of $\operatorname{det} S_{V}(\lambda)$, and of $-\lambda_{0}$, as a pole of the cut-off resolvent $\mathcal{R}_{V}(\lambda)$, coincide. Consequently, the function

$$
f_{V}(\lambda) \equiv \operatorname{det} S_{V}(\lambda),
$$

is holomorphic for $\operatorname{Im} \lambda>c_{0}\left\langle\|V\|_{L^{\infty}}\right\rangle$, and well-defined for $\operatorname{Im} \lambda \geq 0$ with finitely many poles. Hence, the problem of estimating the number of zeros of $f_{V}(\lambda)$ in the upper half plane is the same as estimating the number of resonances in the lower half plane.

This is facilitated in the odd dimensional case by the well-known representation of $f_{V}(\lambda)$ in terms of canonical products. Let $G(\lambda ; p)$ be defined for integer $p \geq 1$, by

$$
G(\lambda ; p)=(1-\lambda) e^{\lambda+\lambda^{2} / 2+\cdots+\lambda^{p} / p},
$$

and define

$$
P(\lambda)=\Pi_{\lambda_{j} \in \mathcal{R}_{V}, \lambda_{j} \neq 0} G\left(\lambda / \lambda_{j} ; d-1\right) .
$$


Then the function $f_{V}(\lambda)$ may be written as

$$
f_{V}(\lambda)=\alpha e^{i g(\lambda)} \frac{P(-\lambda)}{P(\lambda)},
$$

where $g(\lambda)$ is a polynomial of order at most $d$. Careful study of the scattering matrix and the upper bound of section 1.1.1 may be used to show that $f_{V}(\lambda)$ is of order at most $d$ in the half-plane $\operatorname{Im} \lambda>c_{0}\left\langle\|V\|_{\infty}\right\rangle$, see [44]. It is the representation (19) that is not available in the even dimensional case. This facilitates the construction of a plurisubharmonic function (see sections 44 5) in the odd dimensional case as done in [3, 5.

\section{Proof of Generic Behavior}

The proof of Theorem 1 consists of two components. The first is the $G_{\delta^{-}}$-property of the set of all potentials in $L_{0}^{\infty}(K ; F)$, for $F=\mathbb{R}$ or $F=\mathbb{C}$ separately, and $K \subset \mathbb{R}^{d}$ a compact set with nonempty interior, with the correct order of growth. This is fairly easy to prove. The second is the proof that this $G_{\delta}$-set is dense. The density argument is the difficult part.

Many of the statements in the proof of the $G_{\delta}$-property do not depend on whether $d$ is even or odd. We write $f_{V, m}(\lambda)$ with the understanding that $m \in \mathbb{Z}^{*}$ for $d$ even, and $m=-1$ for $d$ odd. We use the same convention for the counting function $n_{V, m}(r)$, so that $n_{V,-1}(r)=n_{V}(r)$.

We first need a Jensen-type theorem that relates the order of growth of the zero counting function $n_{V, m}(r)$ to the asymptotic behavior of $f_{V, m}(\lambda)$. In general, we consider a function $h$ holomorphic in the set $\{\lambda \in \mathbb{C}:|\lambda| \geq$ $R \geq 0, \operatorname{Im} \lambda \geq 0\}$. For $r>R$, we define $n_{+, R}(h, r)$ to be the number of zeros of $h$, counted with multiplicities, in the closed upper half plane with norm between $R$ and $r$, inclusive.

Lemma 1. Let $R>0$, let $h$ be holomorphic in $\{\lambda \in \mathbb{C}|| \lambda \mid \geq R>0, \operatorname{Im} \lambda \geq$ $0\}$. Suppose, in addition, that $h$ has only finitely many zeros on the real axis. Suppose that for some $p>0$, and for some $\epsilon>0$, we have

$$
\int_{R}^{r}\left|\frac{h^{\prime}(s)}{h(s)}\right| d s=\mathcal{O}\left(r^{p-\epsilon}\right) \text { and } \int_{-r}^{-R}\left|\frac{h^{\prime}(s)}{h(s)}\right| d s=\mathcal{O}\left(r^{p-\epsilon}\right) .
$$

Then $n_{+, R}(h, r)$ has order $p$ if and only if

$$
\lim \sup _{r \rightarrow \infty} \frac{\log \int_{0}^{\pi} \log \left|h\left(r e^{i \theta}\right)\right| d \theta}{\log r}=p .
$$

We will apply this lemma with $h$ taken to be $f_{V, m}$. For positive constants $N, M, q>0$ and $j>2 N$, we define subsets of $L_{0}^{\infty}(K ; F)$ by

$$
\begin{aligned}
A_{m}(N, M, q, j) \equiv & \left\{V \in L_{0}^{\infty}(K ; F):\left\langle\|V\|_{L^{\infty}}\right\rangle \leq N,\right. \\
& \int_{0}^{\pi} \log \left|f_{V, m}\left(r e^{i \theta}\right)\right| d \theta \leq M r^{q}, \\
& \text { for } 2 N \leq r \leq j\} .
\end{aligned}
$$


Lemma 2. The set $A_{m}(N, M, q, j) \subset L_{0}^{\infty}(K ; F)$ is closed.

The proof is a continuity argument in the potential $V$. Suppose $V_{k} \in$ $A_{m}(N, M, q, j)$ converges to $V$ in the $L^{\infty}$-norm. We show that the corresponding functions $f_{m}(z)$ converge using the basic bound [29]

$$
|\operatorname{det}(1+A)-\operatorname{det}(1+B)| \leq\|A-B\|_{1} e^{\|A\|_{1}+\|B\|_{1}+1},
$$

with $A=\left(I+V_{j} R_{0}(\lambda) \chi\right)^{-1} V_{j} T(\lambda) \chi$ and $B=\left(I+V R_{0}(\lambda) \chi\right)^{-1} V T(\lambda) \chi$.

In the next step, we characterize those $V \in L_{0}^{\infty}(K ; F)$ for which the resonance counting function exponent is strictly less than the dimension $d$. For $N, M, q>0$, let

$$
B_{m}(N, M, q)=\bigcap_{j \geq 2 N} A_{m}(N, M, q, j) .
$$

Note that $B_{m}(N, M, q)$ is closed by Lemma 2

Lemma 3. Let $V \in L_{0}^{\infty}(K ; F)$, with

$$
\limsup _{r \rightarrow \infty} \frac{\log n_{V, m}(r)}{\log r}<d .
$$

Then there exist $N, M \in \mathbb{N}, l \in \mathbb{N}$, such that $V \in B_{m}(N, M, d-1 / l)$.

The proof of this lemma uses Lemma 1 with $h=f_{V, m}$. Condition (23) implies that

$$
\limsup _{r \rightarrow \infty} \frac{\log \int_{0}^{\pi} \log \left|f_{V, m}\left(r e^{i \theta}\right)\right| d \theta}{\log r}=p<d .
$$

From this it follows that there are constants $p^{\prime}, M>0$, with $p \leq p^{\prime}<d$, so that

$$
\int_{0}^{\pi} \log \left|f_{V, m}\left(r e^{i \theta}\right)\right| d \theta \leq M r^{p^{\prime}}
$$

when $r \geq c_{0}\left\langle\|V\|_{L^{\infty}}\right\rangle$. The lemma then follows by choosing $\ell \in \mathbb{N}$ so that $p^{\prime} \leq d-1 / \ell$.

Lemma 4. For $d \geq 2$ even and $m \in \mathbb{Z}^{*}$ or $d \geq 1$ odd and $m=-1$, the sets

$$
\mathcal{M}_{m}=\left\{V \in L_{0}^{\infty}(K ; F): \lim \sup _{r \rightarrow \infty} \frac{\log n_{V, m}(r)}{\log r}=d\right\},
$$

are $G_{\delta}$-sets. Furthermore, for $d \geq 2$ even, the set

$$
\mathcal{M}=\bigcap_{m \in \mathbb{Z}^{*}} \mathcal{M}_{m}
$$

is also a $G_{\delta}$-set.

Proof. Lemma 3 shows that the complement of $\mathcal{M}_{m}$ is contained in

$$
\bigcup_{(N, M, l) \in \mathbb{N}^{3}} B_{m}(N, M, d-1 / l),
$$

which is an $F_{\sigma}$ set since it is a countable union of closed sets. By Lemma 1 , if $V \in \mathcal{M}_{m}$, then $V \notin B_{m}(N, M, d-1 / l)$ for any $N, M, l \in \mathbb{N}$. Thus $\mathcal{M}_{m}$ is the complement of an $F_{\sigma}$ set. 
Theorem 1 follows from Lemmas 10-4 and the density argument presented here that relies on sections 46 .

Proof of Theorem 1. Since Lemma 4 shows that $\mathcal{M}_{m}$ is a $G_{\delta}$ set, we need only show that each is dense in $L_{0}^{\infty}(K ; F)$. To do this, we follow the proof of [3. Corollary 1.3] with appropriate modifications. Let $V_{0} \in L_{0}^{\infty}(K ; F)$ and let $\epsilon>0$. By Theorem 2, proved in section 6, we may choose a nonzero, real-valued, spherically symmetric $V_{1} \in L_{0}^{\infty}(K ; \mathbb{R})$ so that $V_{1} \in \mathcal{M}_{m}$, for $m \in \mathbb{Z}^{*}$. We consider the holomorphic function $V(z)=V(z, x)=z V_{1}(x)+$ $(1-z) V_{0}(x)$, for $z \in \mathbb{C}$. This function satisfies Assumptions (V) of section [5, with $V(1)=V_{1}$ and $V(0)=V_{0}$. Thus, by [3, Theorem 1.1], for $d$ odd, and by [6. Theorem 3.8], for $d$ even, there exists a pluripolar set $E_{m} \subset \mathbb{C}$ (see Definition 4.2), so that for $z \in \mathbb{C} \backslash E_{m}$, we have

$$
\limsup _{r \rightarrow \infty} \frac{\log n_{V(z), m}(r)}{\log r}=d .
$$

If we set $E=\cup_{m \in \mathbb{Z}^{*}} E_{m}$, the set $E \subset \mathbb{C}$ is also pluripolar [11, Proposition 1.37]. Since $E \cap \mathbb{R} \subset \mathbb{R}$ has Lebesgue measure 0 (e.g. [20, Section 12.2]), we may find $z_{0} \in \mathbb{R}, z_{0} \notin E$, with $\left|z_{0}\right|<\epsilon /\left(1+\left\|V_{0}\right\|_{L^{\infty}}+\left\|V_{1}\right\|_{L^{\infty}}\right)$. Then $V\left(z_{0}\right) \in \mathcal{M}_{m}$ for all $m \in \mathbb{Z}^{*}$, and $\left\|V\left(z_{0}\right)-V_{0}\right\|_{L^{\infty}}<\epsilon$. Moreover, if $V_{0}$ is real-valued, so is $V\left(z_{0}\right)$.

\section{Plunisubharmonic Functions and Pluripolar Sets}

A key role is played in the density part of the proof of Theorem 1 by the theory of plurisubharmonic functions. Plurisubharmonic functions were first used in the study of the resonance counting function in [3]. A basic reference is the book by Lelong and Gruman [11. We refer to an open connected set $\Omega \subset \mathbb{C}^{k}$ as a domain. For two domains $\Omega^{\prime}$ and $\Omega$, we use the notation $\Omega^{\prime} \Subset \Omega$ if $\overline{\Omega^{\prime}}$ is compact and $\overline{\Omega^{\prime}} \subset \Omega$.

Definition 4.1. A real-valued function $\phi(z)$ taking values in $[-\infty, \infty)$ is plurisubharmonic in a domain $\Omega \subset \mathbb{C}^{k}$, and we write $\phi \in P S H(\Omega)$, if:

- $\phi$ is upper semicontinuous and $\phi \not \equiv-\infty$;

- for every $z \in \Omega$, for every $w \in \mathbb{C}^{k}$, and every $r>0$ such that $\{z+u w:|u| \leq r, u \in \mathbb{C}\} \subset \Omega$ we have

$$
\phi(z) \leq \frac{1}{2 \pi} \int_{0}^{2 \pi} \phi\left(z+r e^{i \theta} w\right) d \theta .
$$

If the dimension $k=1$, then this is just the definition of a subharmonic function. A basic example of a PSH function is $\phi_{f}(z)=\log |f(z)|$ where $f$ is holomorphic in a domain $\Omega$. If $f\left(z_{0}\right)=0$, for some $z_{0} \in \Omega$, then $\phi\left(z_{0}\right)=-\infty$. This shows that there is a connection between the behavior of this PSH function $\phi_{f}(z)$ on $\Omega$ and the zeros of $f$ on $\Omega$. The points where a nontrivial PSH function takes the value $-\infty$ are special and rare. 
Definition 4.2. A set $E \subset \mathbb{C}^{k}$ is pluripolar if for each $a \in E$ there is a neighborhood $V_{a}$ containing a and a function $\phi_{a} \in P S H\left(V_{a}\right)$ such that $E \cap V_{a} \subset\left\{z \in V_{a}: \phi_{a}(z)=-\infty\right\}$.

Pluripolar sets have many properties. Of course, if $E \subset \mathbb{C}$ is pluripolar, then the two-dimensional Lebesgue measure of $E$ is zero. Moreover, the one-dimensional Lebesgue measure of $E \cap \mathbb{R}$ is also zero.

The order of growth $\rho$ of a PSH function $\phi$ on the complex plane $\mathbb{C}$ is defined to be

$$
\rho \equiv \limsup _{r \rightarrow \infty} \frac{\log \sup _{0 \leq \theta \leq 2 \pi}\left|\phi\left(r e^{i \theta}\right)\right|}{\log r},
$$

when it exists and is finite. We consider PSH functions on domains of the form $\Omega \times \mathbb{C}$, with $\Omega \subset \mathbb{C}^{k}, k \geq 1$. For fixed $z^{\prime} \in \Omega$, let $\rho\left(z^{\prime}\right)$ be the order of growth of $u \in \mathbb{C} \rightarrow \phi\left(z^{\prime}, u\right)$, where $z=\left(z^{\prime}, u\right) \in \Omega \times \mathbb{C}$.

We use two main results in the theory of PSH functions in order to analyze PSH functions on domains of the form $\Omega \times \mathbb{C}$ and their parameterized order of growth $\rho\left(z^{\prime}\right)$. These are presented in the textbook by Lelong and Gruman [11]. The first result ([11, Proposition 1.40]) is that for $\Omega^{\prime} \Subset \Omega$, there is a sequence of negative PSH functions $\psi_{n}$ on $\Omega^{\prime}$ so that $\left[\rho\left(z^{\prime}\right)\right]^{-1}=$ $-\lim \sup _{n \rightarrow \infty} \psi_{n}\left(z^{\prime}\right)$, where $z^{\prime} \in \Omega^{\prime}$. The second result ([11, Proposition 1.39]) concerns a sequence $\psi_{n}$ of PSH functions uniformly bounded above on $\Omega \subset \mathbb{C}^{k}$ with $\limsup _{n \rightarrow \infty} \psi_{n} \leq 0$. If there is one $z_{0} \in \Omega$ such that $\limsup _{n \rightarrow \infty} \psi_{n}\left(z_{0}\right)=0$, then the set $\left\{z \in \Omega \mid \limsup _{n \rightarrow \infty} \psi_{n}(z)<0\right\}$ is pluripolar in $\Omega$.

In our application of these results, we will show that the order of growth of a certain PSH function is at most $d$ so that, applying the first result sketched above, $\lim \sup _{n \rightarrow \infty}\left(\psi_{n}\left(z^{\prime}\right)+1 / d\right) \leq 0$. We will find a $z_{0}$ for which the limit superior is exactly zero, so that the second result mentioned above implies that the limit superior is zero for all $z \in \Omega^{\prime}$ except for a pluripolar set. This is the essence of the density argument. We next turn to the construction of the appropriate PSH functions.

\section{PARAmeterized Potentials AND the ORDER OF GROWTH}

The theory of plurisubharmonic functions is applied to the resonance counting problem through the introduction of families of holomorphic potentials $V(x ; z)$ with $z \in \Omega \subset \mathbb{C}^{k}$. The main result is roughly the following: If there is a point $z_{0} \in \Omega$ for which the order of growth $\rho_{V\left(z_{0}\right)}$ for the resonance counting function for the potential $V\left(z_{0}\right)$ is maximal (so upper and lower bounds on the resonance counting function of the same order $d$ are required), then there is a pluripolar set $E \subset \Omega$ so that the order of growth is maximal for all potentials $V(z)$ with $z \in \Omega \backslash E$. This provides the proof of density as in section 3 , 
In order to achieve this result, one has to construct a plurisubharmonic function $(\mathrm{PSH})$ that reflects the order of growth of the resonance counting function for the family of potentials $V(z ; x)$. Let $\Omega \subset \mathbb{C}^{k}$ be an open connected set. Let $V(z) \equiv V(z ; x)$ be a family of potentials satisfying

- For $z \in \Omega, V(z, \cdot) \in L_{0}^{\infty}\left(\mathbb{R}^{d}\right)$.

- The function $V(z, x)$ is holomorphic in $z \in \Omega$.

- There is a compact set $K_{1} \subset \mathbb{R}^{d}$ so that for $z \in \Omega, V(z, x)=0$ if $x \in \mathbb{R}^{d} \backslash K_{1}$.

We will refer to these properties as Assumptions ( $V)$. We are interested in the resonance counting functions for potentials satisfying Assumptions (V). For such a potential $V(z)$, we define, in analogy to (14), the function

$$
f_{m}(z, \lambda)=\operatorname{det}\left(I-m\left(I+V(z) R_{0}(\lambda) \chi\right)^{-1} V(z) T(\lambda) \chi\right)
$$

for $\lambda \in \Lambda_{0}$.

In order to apply the theory of [11, we construct a plurisubharmonic function $M(z, u)$ on $\Omega^{\prime} \times \mathbb{C}$, for any $\Omega^{\prime} \Subset \Omega$, from (27). This function has the property that $M(z,|u|)$ is a positive, monotone increasing, function of $|u|$, for any $z \in \Omega$. Recalling the holomorphicity of $f_{m}$, we define, for any $\epsilon>0$, a function $g_{m, \epsilon}(z, u)$ by

$$
g_{m, \epsilon}(z, u)=\int_{0}^{\pi} \log \left|f_{m}\left(z, u e^{i \theta}\right)\right| d \theta+\log \left|e^{u^{d-\epsilon}}\right| .
$$

For $\Omega^{\prime} \Subset \Omega$, we define the constant $V_{M, \Omega^{\prime}}=\max _{z \in \Omega^{\prime}}\|V(z, \cdot)\|_{L^{\infty}}$. The function $g_{m, \epsilon}(z, u)$ is PSH on the strip-like domain of the form $\Omega^{\prime} \times U_{\Omega^{\prime}}$, where $\Omega^{\prime} \Subset \Omega$ and $U_{\Omega^{\prime}} \subset \mathbb{C}$ is given by

$$
U_{\Omega^{\prime}}=\left\{u \in \mathbb{C}|| \operatorname{Im} u\left|<2, \operatorname{Re} u>c_{0} V_{M, \Omega^{\prime}},\right| \arg u \mid<\pi / 4\right\} .
$$

The second term in (28) allows us to control the location of the maximum of $g_{m, \epsilon}$ on the intersection of the domain $\Omega^{\prime} \times U_{\Omega^{\prime}}$ with the arcs $|u|=r$.

Starting with $g_{m, \epsilon}$ defined in (28), we construct a PSH function on $\Omega^{\prime} \times \mathbb{C}$, for any $\Omega^{\prime} \Subset \Omega$, through a series of extensions that we briefly summarize here. First, one proves that there exists $r_{m}>0$ so that for $r>r_{m}\left\langle V_{M, \Omega^{\prime}}\right)^{1 /(1-\epsilon)}>$ 0 ,

$$
\max _{\substack{|\operatorname{Im} u| \leq 1, \operatorname{Re} u>0 \\|u|=r, u \in U_{\Omega^{\prime}}}} g_{m, \epsilon}(z, u)>\max _{\substack{\operatorname{Im} u= \pm 1, \operatorname{Re} u>0 \\|u|=r, u \in U_{\Omega^{\prime}}}} g_{m, \epsilon}(z, u),
$$

for $z \in \Omega^{\prime}$. Second, we note that by using inequality (30) we have

$$
\tilde{M}_{m, \epsilon, \Omega^{\prime}}(z, w)=\max _{\substack{|\operatorname{Im} u| \leq 1,|\arg u| \leq \pi / 4 \\ r_{m}\left(\left\langle V_{M, \Omega^{\prime}}\right\rangle^{1 /(1-\epsilon)}+1\right) \leq|u| \leq|w|}} g_{m, \epsilon}(z, u),
$$

is plurisubharmonic on $\Omega^{\prime} \times\left\{w \in \mathbb{C}: \quad \tilde{r}_{m, \epsilon}\left(\Omega^{\prime}, V\right)<|w|\right\}$, for a suitable constant $\tilde{r}_{m, \epsilon}\left(\Omega^{\prime}, V\right)>0$. Finally, we extend this to a PSH function on 
$\Omega^{\prime} \times \mathbb{C}$. We prove that the function

$M_{m, \epsilon, \Omega^{\prime}}(z, w)= \begin{cases}\max \left(1, \tilde{M}_{m, \epsilon, \Omega^{\prime}}\left(z, \tilde{r}_{m}\left(\Omega^{\prime}, V\right)+1\right),\right. & \text { if }|w| \leq \tilde{r}_{m}\left(\Omega^{\prime}, V\right)+1 \\ \max \left(1, \tilde{M}_{m, \epsilon, \Omega^{\prime}}(z, w)\right), & \text { if }|w| \geq \tilde{r}_{m}\left(\Omega^{\prime}, V\right),\end{cases}$

is plurisubharmonic on $\Omega^{\prime} \times \mathbb{C}$.

Note that the dependence of $M_{m, \epsilon, \Omega^{\prime}}(z, w)$ on $w$ is only through the norm $|w|$ and is a monotone increasing function of $|w|$. The following lemma demonstrates the relationship between the order of $n_{V(z)}(r)$ and the order of $r \mapsto M_{m, \epsilon, \Omega^{\prime}}(z, r)$ for any $z \in \Omega^{\prime}$ fixed.

Lemma 5. Let $\Omega^{\prime} \Subset \Omega$ and let $\rho_{m, \epsilon, \Omega^{\prime}}(z)$ be the order of $r \rightarrow M_{m, \epsilon, \Omega^{\prime}}(z, r)$. We then have

$$
\rho_{m, \epsilon, \Omega^{\prime}}(z)=\max \left(d-\epsilon, \text { order of } n_{V(z), m}(r)\right)
$$

for $z \in \Omega^{\prime}$, where, as above, $n_{V(z), m}(r)$ is the number of resonances of $H_{V(z)}$ on $\Lambda_{m}, m \in \mathbb{Z}^{*}$ of norm at most $r>0$.

The main result of this construction, and the general results for PSH functions presented in section 4 , is the following theorem. The theorem assets that except for a small set of $z \in \Omega$, the order of growth of $n_{V(z), m}(r)$ is $d$ provided it is bounded by $d$ for $z \in \Omega$ and provided that it obtains this value for at least one point in $\Omega$.

Theorem 3. Let $\Omega \subset \mathbb{C}^{d^{\prime}}$ be an open connected set, let $m \in \mathbb{Z}$, and let $V(z, x)$ satisfy the assumptions $(V)$. If for some $z_{m} \in \Omega$, the function $n_{V\left(z_{m}\right), m}(r)$ has order $d$, then there is a pluripolar set $E_{m} \subset \Omega$ such that $n_{V(z), m}(r)$ has order $d$ for $z \in \Omega \backslash E_{m}$. Moreover, if for each $m \in \mathbb{Z}^{*}$, there is a $z_{m}$ such that $n_{V\left(z_{m}\right), m}(r)$ has order $d$, then there is a pluripolar set $E$ such that for every $m \in \mathbb{Z}^{*}$, the function $n_{V(z), m}(r)$ has order $d$ for $z \in \Omega \backslash E$.

\section{LOWER BOUNDS FOR SOME SPHERICALLY SYMMETRIC POTENTIALS}

We compute a lower bound on the number of resonances for $H_{V}$ when $V(x)=V_{0} \chi_{B_{R}(0)}(x)$, with $V_{0}>0$, using separation of variables and uniform asymptotics of Bessel and Hankel functions due to Olver [17, 18, 19]. This method works for $d$ even or odd, thus providing an alternate to using the more precise asymptotic result of Zworski [43] for $d$ odd, as was done in [3, 5]. Because of the spherical symmetry of $V$, we can reduce the Hamiltonian $H_{V}$ to a direct sum of Hamiltonians $H_{\ell}$, for $\ell=0,1,2, \ldots$ acting on $L^{2}\left(\mathbb{R}^{+}\right)$. An important parameter is $\nu \equiv \ell+(d-2) / 2$ that is an integer for $d$ even and half an odd integer for $d \geq 3$ odd. We construct the Green's function on the physical sheet $\Lambda_{0}$ for the reduced Hamiltonian $H_{\ell}$. We let $V(r)=V_{0} \chi_{[0,1]}(r)$, with $V_{0}>0$, and we let $\Sigma(\lambda) \equiv\left(\lambda^{2}-V_{0}\right)^{1 / 2}$, where the square root is defined so that this function has branch cuts $\left(-\infty,-V_{0}^{1 / 2}\right] \cup\left[V_{0}^{1 / 2}, \infty\right)$. Because of 
the simple nature of the potential $V(r)$, the reduced ordinary differential equation for $0<r<1$ is

$$
-\psi_{\nu}^{\prime \prime}-\frac{(d-1)}{r} \psi_{\nu}^{\prime}+\frac{\ell(\ell+d-2)}{r^{2}} \psi_{\nu}=\Sigma(\lambda)^{2} \psi_{\nu},
$$

and for $r>1$, the solution $\psi_{\nu}$ satisfies the free equation

$$
-\psi_{\nu}^{\prime \prime}-\frac{(d-1)}{r} \psi_{\nu}^{\prime}+\frac{\ell(\ell+d-2)}{r^{2}} \psi_{\nu}=\lambda^{2} \psi_{\nu}
$$

We use the ordinary Bessel and Hankel functions of the first kind, denoted by $J_{\nu}$ and $H_{\nu}^{(1)}$, and their spherical counterparts $j_{\nu}$ and $h_{\nu}^{(1)}$. We refer to [17] for complete definitions and properties. We choose two linearly independent solutions, $\phi_{\nu}$ and $\psi_{\nu}$ of (34)-(35) so that $\phi_{\nu}(r=0 ; \lambda)=0$ and $\psi_{\nu}(r ; \lambda)=$ $h_{\nu}(\lambda r)$ for $r>1$. The Green's function has the form

$$
G_{\nu}\left(r, r^{\prime} ; \lambda\right)=\frac{1}{W_{\nu}(\lambda)}\left\{\begin{array}{l}
\phi_{\nu}(r ; \lambda) \psi_{\nu}\left(r^{\prime} ; \lambda\right), \quad r<r^{\prime} \\
\phi_{\nu}\left(r^{\prime} ; \lambda\right) \psi_{\nu}(r ; \lambda), \quad r>r^{\prime}
\end{array}\right.
$$

where the Wronskian $W_{\nu}(\lambda)$, evaluated at $r=1$, is given by

$$
W_{\nu}(\lambda)=\Sigma(\lambda) j_{\nu}^{\prime}(\Sigma(\lambda)) h_{\nu}^{(1)}(\lambda)-\lambda j_{\nu}(\Sigma(\lambda)) h_{\nu}^{(1)^{\prime}}(\lambda) .
$$

It follows that $\lambda_{0} \in \Lambda_{m}, m \neq 0$, for $d$ even, or $\lambda_{0} \in \Lambda_{-1} \equiv \mathbb{C}^{-}$, if $d$ is odd, is a resonance if it satisfies the following condition:

$$
\Sigma\left(\lambda_{0}\right) J_{\nu}^{\prime}\left(\Sigma\left(\lambda_{0}\right)\right) H_{\nu}^{(1)}\left(\lambda_{0}\right)-\lambda_{0} J_{\nu}\left(\Sigma\left(\lambda_{0}\right)\right) H_{\nu}^{(1)^{\prime}}\left(\lambda_{0}\right)=0, \nu=\ell+(d-2) / 2,
$$

where we used the definitions of the spherical functions in terms of the standard functions.

In order to study the defining equation (38) on $\Lambda_{m}$, we define a function $F_{m}^{(\nu)}(\lambda)$ on $\Lambda_{0}$ by

$$
F_{m}^{(\nu)}(\lambda)=\Sigma(\lambda) J_{\nu}^{\prime}(\Sigma(\lambda)) H_{\nu}^{(1)}\left(e^{i m \pi} \lambda\right)-e^{i m \pi} \lambda J_{\nu}(\Sigma(\lambda)) H_{\nu}^{(1)^{\prime}}\left(e^{i m \pi} \lambda\right),
$$

using the fact that $\Sigma\left(e^{i m \pi} \lambda\right)=\Sigma(\lambda)$, for $m \in \mathbb{Z}$. It follows from the fundamental equation (38) that the zeros of $F_{m}^{(\nu)}(\lambda)$ on $\Lambda_{0}$ correspond to the resonances of the one-dimensional Schrödinger operator $H_{\ell}$ on the sheet $\Lambda_{m}$, for $|m| \geq 1$. For $d$ odd, there are only two independent functions corresponding to $m=0$ and $m=-1$. As $m=0$ corresponds to the physical sheet, there are no resonances, and because $V_{0}>0$, there are no eigenvalues. For $m=-1$, the resonances are the zeros of $F_{1}^{(\nu)}(\lambda)$ for $0<\arg \lambda<\pi$ and $\nu$ a half-odd integer. We prove that this number is bounded below by $C_{-1} r^{d}$, with $C_{-1}>0$. For $d$ even, we will prove that the number of zeros is bounded below by $C_{m} r^{d}$ on each nonphysical $m \neq 0$, for some constant $C_{m}>0$.

The zeros of $F_{m}^{(\nu)}(\lambda), \lambda \in \Lambda_{0}$, are studied using the uniform asymptotic expansions of the Bessel and Hankel functions proved by Olver [18, 19]. A similar method was used by Stefanov [33. It is convenient to define new variables $z=\lambda / \nu$ and $\tilde{z}(z)=\left(z^{2}-\nu^{-2} V_{0}\right)^{1 / 2}$. The formulas for the analytic continuation of Bessel and Hankel functions [17, chapter 7] allow one to 
reduce the question of the zeros of $F_{m}^{(\nu)}(\lambda), \lambda \in \Lambda_{0}$, to considering those $\lambda \in \Lambda_{0}$ for which

$$
F_{0}^{(\nu)}(\nu z)=2 m G_{0}^{(\nu)}(\nu z)
$$

where, from (39),

$$
F_{0}^{(\nu)}(\nu z)=\nu \tilde{z} J_{\nu}^{\prime}(\nu \tilde{z}) H_{\nu}^{(1)}(\nu z)-\nu z J_{\nu}(\nu \tilde{z}) H_{\nu}^{(1)^{\prime}}(\nu z)
$$

and we define

$$
G_{0}^{(\nu)}(\nu z) \equiv \nu \tilde{z} J_{\nu}^{\prime}(\nu \tilde{z}) J_{\nu}(\nu z)-\nu z J_{\nu}(\nu \tilde{z}) J_{\nu}^{\prime}(\nu z) .
$$

It is sufficient for the lower bound to prove that for any $\nu<r$, for $\nu>\nu_{0}$ and $r>>0$ sufficiently large, that there are at least $\nu\left(1-\epsilon_{1}\right), \epsilon_{1}>0$ small, solutions of the equation (40) in the half-disk $\operatorname{Im} \lambda>0$ and $|\lambda| \leq r$, uniformly in $r$ and $\nu$.

A special role in the uniform asymptotics of the Bessel and Hankel functions is played by the compact, eye-shaped region $K$ in the complex plane defined as follows. Let $t_{0}$ be the positive root of $t=\operatorname{coth} t$, so $t_{0} \sim 1.19967864 \ldots$ The region $K$ is the symmetric region in the neighborhood of the origin bounded in $\mathbb{C}^{+}$by the curve

$$
z= \pm\left(t \operatorname{coth} t-t^{2}\right)^{1 / 2}+i\left(t^{2}-t \tanh t\right)^{1 / 2}, 0 \leq t \leq t_{0}
$$

intercepting the real axis at \pm 1 and intercepting the imaginary axis at $i z_{0}$, where $z_{0}=\left(t_{0}^{2}-1\right)^{1 / 2} \sim 0.66274 \ldots$. The region $K$ is bounded by the conjugate curve in the lower half-plane. To prove that there are at least $\nu\left(1-\epsilon_{1}\right)$ zeros of $F_{m}^{(\nu)}(\lambda)$ near the upper boundary of the eye-shaped region $\nu K$, we concentrate a small region $\Omega_{1, \epsilon} \subset \mathbb{C}$, in the $z$ variable, near the upper boundary of $K$, defined, for fixed $\epsilon>0$, by $\Omega_{1, \epsilon}=\left\{z \in \mathbb{C}^{+}\right.$: dist $\left(z, \partial K^{+}\right)<$ $\epsilon\} \cap\left\{z \in \mathbb{C}^{+}:|z+1|>\epsilon\right.$ and $\left.|z-1|>\epsilon\right\}$.

We computed the uniform asymptotics of each term in (40). In order to state these, we need a map $z \rightarrow \rho(z)$ defined by

$$
\rho(z) \equiv \log \frac{1+\sqrt{1-z^{2}}}{z}-\sqrt{1-z^{2}}
$$

The uniform asymptotic expansion of $F_{0}^{(\nu)}(\nu z)$ for $z \in \Omega_{1, \epsilon}$, as computed in [6, sections 5 and 6$]$, is

$$
F_{0}^{(\nu)}(\nu z)=\frac{-2 i}{\pi}\left\{1-\frac{1}{\nu}\left[\frac{V_{0}\left(1-z^{2}\right)^{1 / 2}}{2 z^{2}}\right]+\mathcal{O}\left(\frac{1}{\nu^{2}}\right)\right\},
$$

and for $G_{0}^{(\nu)}$, we obtained

$$
G_{0}^{(\nu)}(\nu z)=\frac{e^{-2 \nu \rho}}{2 \pi}\left[\frac{V_{0}}{2 \nu^{2}\left(1-z^{2}\right)}+\mathcal{O}\left(\frac{1}{\nu^{3}}\right)\right],
$$


where the error is uniform for $z \in \Omega_{1, \epsilon}$. Consequently, the condition for zeros on the $m^{t h}$-sheet is that there exists solutions $z \in \Omega_{1, \epsilon}$ to the equation

$$
e^{2 \nu \rho(z)}\left(1+g_{1}(z, \nu)\right)=\frac{i m V_{0}}{4 \nu^{2}}\left(\frac{1}{1-z^{2}}\right)+g_{2}(z, \nu),
$$

where $g_{1}(z, \nu)=\mathcal{O}(1 / \nu)$, and $g_{2}(z, \nu)=\mathcal{O}\left(1 / \nu^{3}\right)$, both uniformly for $z \in$ $\Omega_{1, \epsilon}$. We note that for $V_{0}=0$ there are no solutions to this equation.

We consider (47) as an equation for $\rho$. The variable $\rho$ lies in a set that is the image of $\Omega_{1, \epsilon}$ under the mapping $z \rightarrow \rho$ given in (44). This set contains a neighborhood of an interval of the negative imaginary axis of the form $(-\pi+h(\epsilon),-h(\epsilon)) i \subset(-\pi, 0) i$. We proved that there exists at least $\nu\left(1-\epsilon_{1}\right)$ solutions in a neighborhood of this set, where $\epsilon_{1}=\mathcal{O}(\epsilon)$. We first analyzed the zeros of the function $g(z, \nu)$ defined by

$$
g(z, \nu)=\nu^{2} e^{2 \nu \rho}-\frac{i m V_{0}}{4} .
$$

These can be computed explicitly and have the form

$$
\rho_{k}=\left\{\frac{1}{2 \nu} \log \left(\frac{|m| V_{0}}{4}\right)-\frac{\log \nu}{\nu}\right\}+i \frac{\pi}{\nu}\left[k+\operatorname{sgn}(m) \frac{1}{4}\right], k \in \mathbb{Z} .
$$

Then, using Rouché's Theorem, we proved that in a neighborhood of each zero $\rho_{k}$ of $g(z, \nu)$ with imaginary part in $(-i \pi+i 2 h(\epsilon),-i 2 h(\epsilon))$, there is exactly one solution to (47). Consequently, there are at least $\nu\left(1-\epsilon_{1}\right)$, $\epsilon_{1}=\mathcal{O}(\epsilon)>0$ zeros in a neighborhood of the interval on the negative imaginary axis $(-\pi, 0) i$ for all $\nu>0$ large.

To prove the lower bound, recall that $\nu=l+(d-2) / 2, l \in \mathbb{N}$ and $\epsilon_{1}>0$ is arbitrary. By the symmetry reduction described in the beginning of this section, each zero of $F_{m}^{(\nu)}(\lambda)$ corresponds to a resonance of multiplicity $m(l)$, the dimension of the space of spherical harmonics on $\mathbb{S}^{d-1}$ with eigenvalue $l(l+d-2)$. Since $m(l) \geq c l^{d-2}+\mathcal{O}\left(l^{d-3}\right)$, for some $c>0$, it follows that

$$
n_{m, V}(r) \geq \sum_{\ell=1}^{[r]} \frac{1}{4}(l-(d-2) / 2)\left(c l^{d-2}+\mathcal{O}\left(l^{d-3}\right)\right) \geq C_{m} r^{d}+\mathcal{O}\left(r^{d-1}\right),
$$

for some $C_{m}>0$, depending on $m \in \mathbb{Z}^{*}$. This proves the lower bound on the $m^{t h}$-sheet, $m \in \mathbb{Z}^{*}$. If the potential $V$ is real, the symmetry of the zeros means that the resonances on $\Lambda_{-m}$ are in one-to-one correspondence with those on $\Lambda_{m}$.

\section{Open Problems}

One of the main open problems in this area is the proof of the optimal lower bound $n_{V, m}(r) \geq C_{d, m} r^{d}, C_{d, m}>0$, for nontrivial, real-valued potentials $V \in L_{0}^{\infty}\left(\mathbb{R}^{d}\right)$. Alternately, it would be of interest to construct such a potential whose resonance counting function has order of growth strictly less than $d$ showing that such a lower bound is not possible. The question

of computing an asymptotic expansion for the resonance counting function 
is of great interest but seemingly out of reach at this time. It is not even clear if such an asymptotic expansion should exist.

\section{ACKNOWLEDGEMENTS}

PDH thanks the organizing committee of 35th Journées EDP for the opportunity to present a talk at Evian in June 2008. TJC was partially supported by NSF grant 0500267, MSRI, and an MU Research Leave, and PDH was partially supported by NSF grant 0503784 , during the time some of this work was done.

\section{REFERENCES}

[1] R. Bañuelos, A. Sá Barreto, On the heat trace of Schrödinger operators, Comm. Partial Differential Equations 20 (1995), no. 11-12, 2153-2164.

[2] T. Christiansen, Some lower bounds on the number of resonances in Euclidean scattering, Math. Res. Lett. 6 (1999), no. 2, 203-211.

[3] T. Christiansen, Several complex variables and the distribution of resonances for potential scattering, Commun. Math. Phys 259 (2005), 711-728.

[4] T. Christiansen, Schrödinger operators with complex-valued potentials and no resonances, Duke Math Journal 133, no. 2 (2006), 313-323.

[5] T. Christiansen and P. D. Hislop, The resonance counting function for Schrödinger operators with generic potentials, Math. Research Letters, 12 (6) (2005), 821-826.

[6] T. Christiansen and P. D. Hislop, Maximal order of growth for the resonance counting function for generic potentials in even dimensions, submitted, arXiv:0811.4761v1.

[7] R. Froese, Asymptotic distribution of resonances in one dimension, J. Differential Equations 137 (1997), no. 2, 251-272.

[8] R. Froese, Upper bounds for the resonance counting function of Schrdinger operators in odd dimensions, Canad. J. Math. 50 (1998), no. 3, 538-546.

[9] A. Intissar, A polynomial bound on the number of the scattering poles for a potential in even dimensional spaces $\mathbb{R}^{n}$, Comm. in Partial Diff. Eqns. 11, No. 4 (1986), 367-396.

[10] P. D. Lax and R. S. Phillips, Decaying modes for the wave equation in the exterior of an obstacle, Comm. Pure Appl. Math. 22 (1969), 737-787.

[11] P. Lelong and L. Gruman, Entire functions of several complex variables, Springer Verlag, Berlin, 1986.

[12] G. P. Menzala, T. Schonbek, Scattering frequencies for the wave equation with a potential term, J. Funct. Anal. 55 (1984), 297-322.

[13] R. B. Melrose, Polynomial bounds on the number of scattering poles, J. Funct. Anal. 53 (1983), 287-303.

[14] R. B. Melrose, Geometric scattering theory, Cambridge University Press, 1995.

[15] R. G. Newton, Analytic properties of radial wave functions, J. Math. Phys. 1, no. 4, 319-347 (1960).

[16] H. M. Nussenzveig, The poles of the $S$-matrix of a rectangular potential well or barrier, Nuclear Phys. 11 (1959), 499-521.

[17] F. W. J. Olver, Asymptotics and Special Functions, Academic Press, San Deigo, 1974.

[18] F. W. J. Olver, The asymptotic solution of linear differential equations of the second order for large values of a parameter, Phil. Trans. Royal Soc. London Ser. A 247, 307-327 (1954).

[19] F. W. J. Olver, The asymptotic expansion of Bessel functions of large order, Phil. Trans. Royal Soc. London ser. A 247, 328-368 (1954).

[20] T. Ransford, Potential theory in the complex plane, Cambridge University Press, Cambridge, 1995. 
[21] T. Regge, Analytic properties of the scattering matrix, Il Nuovo Cimento 8 (1958), no. 10, 671-679.

[22] A. Sá Barreto, Remarks on the distribution of resonances in odd dimensional Euclidean scattering, Asymptot. Anal. 27 (2001), no. 2, 161-170.

[23] A. Sá Barreto, Lower bounds for the number of resonances in even dimensional potential scattering, J. Funct. Anal. 169 (1999), 314-323.

[24] A. Sá Barreto, S.-H. Tang, Existence of resonances in even dimensional potential scattering, Commun. Part. Diff. Eqns. 25 (2000), no. 5-6, 1143-1151.

[25] A. Sá Barreto, M. Zworski, Existence of resonances in three dimensions, Comm. Math. Phys. 173 (1995), no. 2, 401-415.

[26] A. Sá Barreto, M. Zworski, Existence of resonances in potential scattering, Comm. Pure Appl. Math. 49 (1996), no. 12, 1271-1280.

[27] N. Shenk, D. Thoe, Resonant states and poles of the scattering matrix for perturbations of $-\Delta$, J. Math. Anal. Appl. 37 (1972), 467-491.

[28] B. Simon, Resonances in one dimension and Fredholm determinants, J. Funct. Anal. 178 (2000), no. 2, 396-420.

[29] B. Simon, Trace Ideals and their Applications, London Mathematical Society Lecture Note Series 35, Cambridge University Press, 1979; second edition, American Mathematical Society, Providence RI, 2005.

[30] B. Simon, Operators with singular continuous spectrum: I. general operators, Ann. Math. 141 (1995), 131-145.

[31] J. Sjöstrand, Geometric bounds on the density of resonances for semiclassical problems, Duke Math. J. 60 (1990), no. 1, 1-57.

[32] J. Sjöstrand, M. Zworski, Complex scaling and the distribution of scattering poles, J. Amer. Math. Soc. 4(1991), no. 4, 729-769.

[33] P. Stefanov, Sharp bounds on the number of the scattering poles, J. Func. Anal., 231 (1) (2006), 111-142.

[34] A. Vasy, Scattering poles for negative potentials, Comm. Partial Differential Equations 22 (1997), no. 1-2, 185-194

[35] G. Vodev, Sharp polynomial bounds on the number of scattering poles for perturbations of the Laplacian, Commun. Math. Phys. 146 (1992), 39-49.

[36] G. Vodev, Sharp bounds on the number of scattering poles in even-dimensional spaces, Duke Math. J. 74 (1) (1994), 1-17.

[37] G. Vodev, Sharp bounds on the number of scattering poles in the two-dimensional case, Math. Nachr. 170 (1994), 287-297.

[38] G. Vodev, Resonances in Euclidean scattering, Cubo Matemática Educacional 3 No. 1, Enero 2001, 319-360.

[39] G. N. Watson, Treatise on the theory of Bessel functions, Cambridge University Press, 1966.

[40] D. Yafaev, Mathematical scattering theory. General theory, translated from the Russian by J. R. Schulenberger, Translations of Mathematical Monographs, 105, American Mathematical Society, Providence, RI, 1992

[41] M. Zworski, Sharp polynomial poles on the number of scattering poles, Duke Math. J. 59 (1989), 311-323.

[42] M. Zworski, Distribution of poles for scattering on the real line, J. Funct. Anal. 73 (1987), 277-296.

[43] M. Zworski, Sharp polynomial bounds on the number of scattering poles of radial potentials, J. Funct. Anal. 82 (1989), 370-403.

[44] M. Zworski, Poisson formulae for resonances, Sminaire sur les Equations aux Dérivées Partielles, 1996-1997, Exp. No. XIII, 14 pp., Ecole Polytech., Palaiseau, 1997 Seminaire Ecole Polytechnique.

[45] M. Zworski, Counting scattering poles, In: Spectral and scattering theory (Sanda, 1992), 301-331, Lectures in Pure and Appl. Math. 161, New York: Dekker, 1994. 
Department of Mathematics , University of Missouri, Columbia, Missouri 65211 USA

E-mail address: tjc@math.missouri.edu

Department of Mathematics, University of Kentucky, Lexington, KenTUCKY 40506-0027, USA

E-mail address: hislop@ms.uky.edu 